\title{
Gratulation! - Die meistzitierten Beiträge im 34. Jahrgang der Psychiatrischen Praxis
}

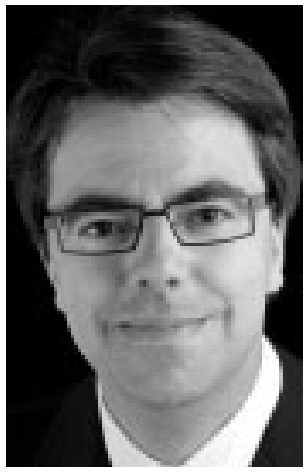

Dr. Oliver H. Günther

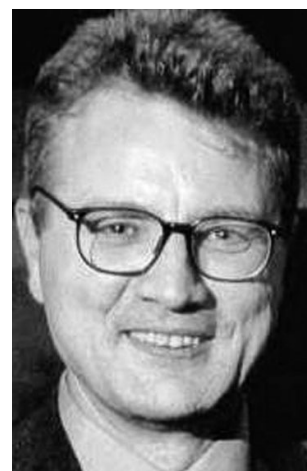

Prof. Hans Joachim Salize

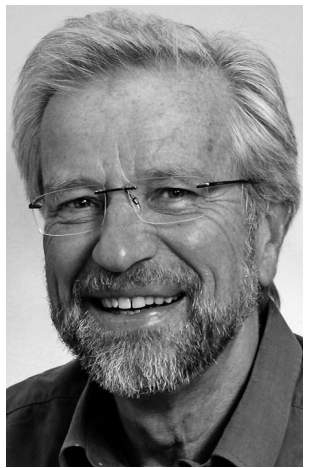

Prof. Wielant Machleidt

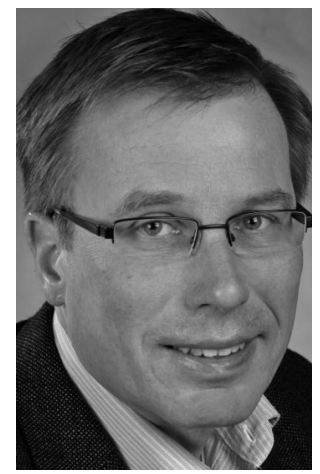

Prof. Dirk Richter

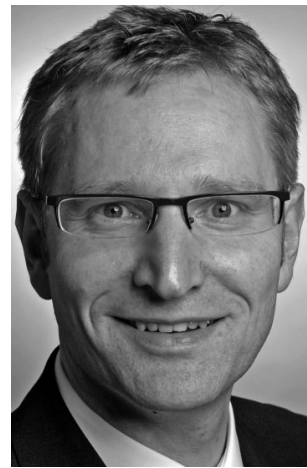

Dr. Hendrik Kluge
Der Beginn des Neuen Jahres, ist eine Gelegenheit zurückzuschauen und Bilanz zu ziehen. Die Psychiatrische Praxis hält Rückschau und kürt auch in diesem Jahr wieder die meistzitierten Beiträge des 34. Jahrgangs der Zeitschrift. Dabei gibt es einen Spitzenreiter und 4 gleichrangige Arbeiten auf dem 2. Platz. Somit werden 5 Arbeiten gekürt, die sich mit klassischen Themen der Sozialpsychiatrie und Versorgungsforschung beschäftigen. Am häufigsten wurde die Arbeit von Oliver $\mathrm{H}$. Günther zur „Krankheitslast von depressiven Erkrankungen in Deutschland“ " [1] zitiert. Die Arbeit entstand in der Arbeitsgruppe von Hans-Helmut König, Professor für Gesundheitsökonomie an der Universität Leipzig und berichtet Ergebnisse aus dem ESEMeD-Projekt (European Study of Epidemiology of Mental Disorders). Dr. Oliver H. Günther ist von Haus aus Psychologe und ist heute als Gesundheitsökonom in der freien Wirtschaft tätig. Die nachfolgenden Arbeiten teilen sich den 2. Platz. Dazu zählt die Arbeit von Hans Joachim Salize, der der Frage nachgeht, ob arbeitsrehabilitative Maßnahmen während stationärpsychiatrischer Behandlung langfristig die Versorgungskosten von Patienten mit Schizophrenie senken [2]. Prof. Hans Joachim Salize ist Leiter der Arbeitsgruppe Versorgungsforschung am Zentralinstitut für Seelische Gesundheit in Mannheim.

Wielant Machleidt legte eine Übersichtsarbeit zur Integration von Migranten in die psychiatrischpsychotherapeutische Versorgung in Deutschland [3] vor. Dabei wird diskutiert, ob die Einrichtung von Spezialdiensten für Migranten oder eine allgemeine Öffnung der bestehenden Versorgungsdienste für diese Personengruppe im Hinblick auf eine optimierte Versorgung zielführend ist. Prof. Wielant Machleidt war Leiter der Abteilung für Sozialpsychiatrie und Psychotherapie an der Medizinischen Hochschule Hannover. Auch als Emeritus ist ihm die transkulturelle Psychiatrie weiter eine Herzensangelegenheit.
Dirk Richter legte eine systematische Literaturübersicht zu Effekten von mitarbeiterbezogenen Trainingsprogrammen zum Aggressionsmanagement in Einrichtungen der Psychiatrie vor [4]. Prof. Dirk Richter, ehemals in der westfälischen Klinik in Münster und im Institut für Soziologie der Westfälischen Wilhelms-Universität Münster, hat heute eine Forschungsprofessur an der Berner Fachhochschule im Fachbereich Gesundheit inne. Hendrik Kluge untersuchte in seiner Arbeit die Auswirkungen struktureller Faktoren auf die Inanspruchnahme sozialpsychiatrischer Dienste [5]. Dr. Hendrik Kluge, ehemals in der Arbeitsgruppe von Thomas Becker und Matthias Angermeyer an der Klinik für Psychiatrie der Universität Leipzig, ist heute freiberuflich als Berater für Personalentwicklung und Konfliktmanagement tätig.

\section{Literatur}

1 Günther OH, Friemel S, Bernert S et al. Die Krankheitslast von depressiven Erkrankungen in Deutschland. Ergebnisse aus dem Projekt European Study of the Epidemiology of Mental Disorders (ESEMeD). Psychiat Prax 2007; 34 (6): 292-301

2 Salize HJ, Schuh C, Krause M et al, KN-SchizophrenieProjektgruppe Arbeitsrehabilitation. Senken arbeitsrehabilitative Maßnahmen während stationärpsychiatrischer Behandlung langfristig die Versorgungskosten von Patienten mit Schizophrenie? Ergebnisse einer kontrollierten Multizenterstudie. Psychiat Prax 2007; 34 (5): 246-248

3 Machleidt W, Behrens K, Ziegenbein M et al. Integration von Migranten in die psychiatrisch-psychotherapeutische Versorgung in Deutschland. Psychiat Prax 2007; 34 (7): 325-331

4 Richter D, Needham I. Effekte von mitarbeiterbezogenen Trainingsprogrammen zum Aggressionsmanagement in Einrichtungen der Psychiatrie und Behindertenhilfe - Systematische Literaturübersicht. Psychiat Prax 2007; 34 (1): 7-14

5 Kluge H, Becker T, Kallert TW et al. Auswirkungen struktureller Faktoren auf die Inanspruchnahme Sozialpsychiatrischer Dienste - eine Mehrebenenanalyse. Psychiat Prax 2007; 34 (1): 20-25
04103 Leipzig

Steffi.Riedel-Heller@medizin. uni-leipzig.de 